\title{
LA AMISTAD ECUATORIANO-CHILENA EN LA TRAYECTORIA DEL PRESIDENTE LUIS CORDERO. MEMORIAS DEL PRIMER CENTENARIO DE LA INDEPENDENCIA
}

\author{
ECUATORIAN-CHILEAN FRIENDSHIP ON THE PATH OF THE \\ PRESIDENT LUIS CORDERO. MEMORIES OF THE \\ INDEPENDENCE'S FIRST CENTENNIAL
}

\author{
maria Cristina Cárdenas Reyes* \\ acardena@ucuenca.edu.ec
}

\section{RESUMEN}

En 1910, Luis Cordero, quien fuera Presidente del Ecuador entre 1892 y 1895, viaja a Chile para representar a su pais en las celebraciones del Primer Centenario de la Independencia del pais del sur. No era la primera ocasión en que su nombre habia estado ligado a Chile. Una tormentosa polémica, en el contexto de la lucha por el poder politico que desembocaria en la Revolución Libenal de 1895 y el gobierno de Eloy Alfaro, le habia atribuido la llamada "venta de la bandera" ecuatoriana al gobierno chileno, generándose un áspero conflicto que conduciria a la renuncia de Cordero en 1895. Eloy Alfaro, ahora Presidente del Ecuador, propone a Luis Cordero asumir la calidad de Ministro Plenipotenciario del Ecuador y asi viajar a Chile con la finalidad de participar en las celebraciones del gobiemo amigo.

Palabras clave: Ecuador, Chile, Luis Cordero, Primer Centenario de La Independencia

\section{ABSTRACT}

In 1910, the former President of Ecuador, Luis Cordero (1892-1895), travels to Chile to represent his country in the celebrations of the First Centenary of the southern country's Independence. It was not the first occasion that his name had been bound to Chile. A stormy polemic, in the context of the fight for the political power that would end in the Liberal Revolution of 1895 and Eloy Alfaro's government, had attributed him the call "sale of the Ecuadorian flag" to the Chilean government, being generated a rough conflict that would lead to Cordero's renouncement in 1895. Eloy Alfaro, now President of Ecuador, intends to Luis Cordero to assume the quality of Plenipotentiary Minister from the Ecuador and this way to travel to Chile with the purpose of participating in the friend government's celebrations:

Keywords: Ecuador, Chile, Luis Cordero, First Centenary of Independence

\section{PRELIMINAR}

En los momentos en que Ecuador y Chile se aprestan a celebrar el bicentenario de la instalación de sus Juntas de Independencia en 1809 y 1810, respectivamente, resulta oportuno resaltar los antiguos lazos de amistad que tradicionalmente han unido a ambos paises, revisando momentos significativos en este enlace que incluye a las principales ciudades del Ecuador: Quito, Cuenca y Guayaquil.

Recordemos que un chileno, Fray Camilo Henriquez, acuñỏ la célebre frase "Quito Luz de América", luego de haber presenciado la violeta represión desplegada en esta ciudad por los sectores realistas el 10 de agosto de 1809 contra los criollos independentistas rebelados. La Junta de Santiago de Chile, organizada el 18 de Septiembre de 1810, invocó al constituirse el ejemplo de la revolución quiteña. Y en 1812, Camilo

\footnotetext{
- Marfa Cristina Cárdenas Reyes es profesoca-investigadorn de la Universidad de Cuenca, Ecuador. Es Doctora en Historia por la Univ, Pablo de Otavide (Sevilla, 2003), Individuo de Nümeto de ta Academia Nacional de Historia, Bcuador, y Miembro Correspondiente de ta Real Academia de ta Historia, Madrid. Ha publicado libros y artículos sobre historia poitica del Ecuador en el pais y el exterior
} 
Henriquez habria hecho grabar esta proclama en una placa colocada en el faro de Valparaiso, según lo informan autores e historiadores ecuatorianos y chilenos, aunque la misma desapareció posteriormentel.

Un personaje poco conocido en el periodo de la Independencia es el gobernador de Cuenca hacia 1820 , Joseph Maria Vásquez de Noboa, nacido en Concepción, Chile, quien habia realizado un rápido ascenso social en la ciudad. Vázquez de Noboa se habia educado en Lima, trasladándose a Cuenca en 1808, donde se dedícó al ejercicio de la abogacia e ingresó por matrimonio en una importante red familiar. Convertido a la tendencia anti realista, es proclamado Jefe Civil y Militar cuando la ciudad se declara República el 3 de noviembre de 1820 .

Hacia 1910, Ecuador y Chile mantenian una dificil relación con el Perú, y la geopolítica había reforzado una vinculación necesaria entre ambos paises. ${ }^{2}$ La Guerra del Pacifico (1879-1884), uno de los procesos históricos más importantes en la historia de Chile durante el siglo XIX, estaba aún muy próxima, en tanto que el Ecuador afrontaba en 1910 una aguda crisis diplomática, precedida por una prolongada disputa limítrofe con el Perú. En mayo de ese año, el Rey de España se habia excusado de arbitrar en este diferendo, ${ }^{3}$ y ambas naciones estaban al borde de un conflicto armado.

Ciertamente, las fricciones entre Chile y Perú y el dilatado conflicto limitrofe de Ecuador y Perú, gravitaron en la intermediación que el entonces Presidente Luis Cordero ${ }^{4}$ habia realizado a favor de Chile en 1894, una acción que incidiria directamente en su renuncia a la presidencia en 1895 , y cuyas circunstancias y contexto se ofrecen más adelante. Cuando Cordero visita Chile en 1910 en representación del gobierno alfarista, los lazos entre ambos países tenían muchos eslabones que aseguraban al ex Presidente una recepción con todos los honores.

Para situar históricamente y contextuar la trayectoria politica de Luis Cordero, conviene revisar brevemente algunas caracteristicas de su tiempo y lugar. Habia nacido en la provincia del Azuay, situada en la región centro-sur de la sierra ecuatoriana. Su capital es Cuenca, ciudad fundada en 1557, importante núcleo comercial en la colonia, capital de uno de los tres departamentos iniciales del estado republicano, y verdadero crisol de doctrinas politicas en el siglo XIX. Es la tercera ciudad del Ecuador, y fue declarada Patrimonio Cultural de la Humanidad por la UNESCO en 1999. El rasgo de profunda regionalidad, antes que regionalismo, signa la trayectoria de los hombres del XIX quienes, como Cordero, trabajaron incansablemente en la formación de la nación ecuatoriana, una tarea sembrada de escollos. El proceso de consolidación del estado ecuatoriano moderno es uno de los más complejos en Hispanoamérica, dado que su configuración entraña el corte de espacios económicos coloniales de carácter regional, diferenciados por sus características administrativas, demográficas, culturales y geográficas. La sociedad ecuatoriana lleva la marca de una reproducción social y económica relativamente autónoma de las regiones, dotadas a su vez de núcleos urbanos generadores de principios organizativos y de intercambio entre si y con el exterior.

La vida politica de Luis Cordero se desenvuelve en función de una posición intermedia entre el autoritarismo conservador de Gabriel Garcia Moreno (1821-1875) ejercido desde Quito, centro administrativo ligado al estado confesional, y el liberalismo radical finisecular que triunfa en Guayaquil con Eloy Alfaro (1842-1912) en 1895. Cordero se adscribió tempranamente a la corriente regional denominada "progresismo azuayo", " una tendencia de orientación católica a favor de la descentralización y que impulsaba firmemente la supremacia de la ley. En la segunda mitad del siglo XIX, el proyecto progresista buscó incesantemente la consolidación de la institucionalidad republicana del país a través de la reactivación de la economía regional y nacional, y usó como instrumento una práctica política de la mediación. Los progresistas ecuatorianos rechazaron permanentemente la etiqueta de "católicos liberales" al estilo europeo con que los conservadores

1. Para reponer la placa extraviada, el 27 de marzo de 2008, y en presencia del Alcalde de Quito. Paco Moncayo, el Presidente del Instituto de Conmemoración Histórica de Chile. Sergio Martinez Baeza, hizo entrega a Valparaiso de una placa coss la frase "Quito Luz de América", instalada en el monumento a Fray Camilo Henriquez en dicha ciudad. Información disponible en wwwecuatorianosenchile.com

2 Sobre la estrecha relación entre ambos pafses en tarno a 1910, ver Barros van Buren, Mario. 1976. La Misión Eastman en el Ecuador, Quito, Casa de la Cultura Ecuatoriana.

3. Para solucionar el diferendo territorial entre Ecuador y Perí, ambos paises habian reactualizado la fórmula del arbitraje del Rey de Espanta, una negociación que se extendió entre 1887 y 1910.

- Identificado por la historiografia ecuatoriana como Luis Cordera para diferenciarlo de sus descendientes homónimos.

5. Ver Cárdenas Reyes, Maria Cristina, 2006. Región y Estado nacional. El progresismo azuayo del siglo XIX (1840-1895), Quito, Academia Nacional de Historia, Ecuador / Universidad Pablo de Olavide

Revista de Historia, año 18, vols. 18-19, 2008-2009, pp. 79-91. 
"ortodoxos" buscaron descalificarlos durante un periodo de áspera lucha politica, y se autodefinieron como "conservadores moderados". Luego de enfrentarse a la presión de definiciones que distorsionaban la institucionalidad del país, implantadas principalmente por los gobiernos de Garcia Moreno entre 1861 y 1875 . optarian en el último cuarto de siglo XIX, y en momentos de extrema tensión definitoria, por supeditar su práctica política a la matriz confesional.

La tendencia progresista de origen regional adquiere reconocimiento nacional y alcanza su máximo nivel doctrinario al obtener la conducción del poder ejecutivo. Dos de sus principales hombres, Antonio Borrero en 1875 y Luis Cordero en 1892, son elegidos para desempeñar la Presidencia de la República, proyectando una relevancia de la politica azuaya que no tendrá parangón en el siglo XX.

\section{ALGUNOS DATOS BIOGRÁFICOS DE LUIS CORDERO (1833-1912)}

Abogado y politico, naturalista, hombre de letras, orador y periodista, nació en la parroquia de Déleg, perteneciente al antiguo cantón de Azogues, por entonces parte de la provincia del Azuay. Al haber disminuido su patrimonio por la guerras de independencia, sus padres, Gregorio Cordero Carrión y Josefa Crespo Rodriguez, ambos vecinos de Cuenca, se habian trasladado al campo para poder sostener a su numerosa familia. Allí, el padre enseñó las primeras letras a sus hijos mediante severos textos de autores sacros españoles. Luis Cordero vivió su niñez en una comarca indigena, por lo que fue bilingủe, español/quichua. Permaneció leal a esta cultura durante toda su vida, contribuyendo a difundirla en diversas publicaciones. A los 14 años realizó sus estudios formales en el Colegio Seminario de San Luis, Cuenca, para luego trasladarse a Quito y cursar estudios superiores en la Universidad Central del Ecuador.

En 1862 se gradúa como doctor en Jurisprudencia, y se incorpora como abogado ante la Corte Superior de Justicia de Cuenca en 1865. En 1867 ingresa por matrimonio en la familia de Miguel Heredia, acaudalado exportador de cascarilla (quina, quinina). 6 Siendo parlamentario en el Congreso de 1867 y de 1868, se retira temporalmente de la politica para dedicarse a la agricultura y el comercio. En los años de mayor auge de la exportación de cascarilla (1850-1895), Luis Cordero fue un próspero hacendado exportador de la misma, buscando mejorar su calidad para aumentar la competitividad en el mercado mundial, donde habia comenzado a ingresar la producción de la India y Jamaica. Por su actividad comercial, estuvo ligado directamente a redes de comerciantes y banqueros guayaquileños, asi como también del exterior.

Fue también uno de aquellos poligrafos que definieron la fisonomia cultural del siglo XIX ecuatoriano, dejando como legado una nutrida producción escrita, Cultivó varios géneros literarios, y se lo considera un destacado precursor de la filologia ecuatoriana con su Diccionario Quichua-Castellano, publicado en Quito en 1895. Gran cultivador de las ciencias naturales y de la botánica, estuvo inmerso en este espiritu que en los siglos XVIII y XIX se relacionaba con un conjunto de valores ligados a la etapa de formación de las nacionalidades: amor a la patria, fe en la ciencia y critica al retraso cientifico, fe religiosa intachable, afán de claridad y sencillez, respeto al cambio paulatino y por lo tanto, negación de las revoluciones.

Tuvo a su cargo diferentes funciones públicas: jefe político de Cuenca (1875), miembro del Gobierno Provisorio de Quito (1883), Presidente del Senado y senador (1885 y 1892) y Ministro Plenipotenciario en Chile (1910). Luego de triunfar en unas reñidas elecciones, sucedió a Antonio Flores Jijón (1833-1915), y ejerció la Presidencia del Ecuador entre 1892 y 1895 . En el ámbíto educativo, se desempeñó como Rector de la Universidad del Azuay (hoy Universidad de Cuenca), de cuya fundación en 1867 habia sido uno de sus principales impulsores. ${ }^{7}$

\section{LA COMPLEJA PRESIDENCIA DE LUIS CORDERO (1892-1895)}

Hacia fines del siglo XIX, tanto liberales como conservadores habian articulado sus fuerzas para impedir una nueva Presidencia de vertiente progresista, cuyo último representante habia sido Antonio Flores

\footnotetext{
6. En el siglo XVII, la clase pudiente del Azuay comenzó a consolidar su fortuna sacando partide de un monopolio comercial de la cascarilla establecido por la corona, y que tenia como principal centro de aprovisionamiento a la Gobernación de Cuenca. La cascarilla fue exportada en abundancia al mercado mundial, siguiendo la ruta Cuenca-Guayaquil-Piura-Lima-España. Hacia 1850 se inicia el ciclo exportador que daria origen a considerables fortunas en la región. Sobre la historia económica de Cuenca. ver Palomeque. Silvia. 1990. Cuenca en el sigio XIX. La articulación de una región. Quita, FL ACSO/Abyu Yala.

7. La Corporación Universitaria del Azuay (1867) se organiza como Universidad del Azuay en 1897. Mantiene esta denominación hasta 1924, ctando adquiere su actual nombre de Universidad de Cuenca.
} 
Jijón durante el período1888-1892. 8 La prensa de la época se referia al nuevo grupo como "la fusión", configurada por la estrecha alianza politica entre las élites de los dos polos de desarrollo en sierra y costa, Quito y Guayaquil.

En 1892, Luis Cordero fue elegido con una clara ventaja sobre su encarnizado oponente Camilo Ponce, cabeza indiscutida de la corriente conservadora de berencia garciana afincada en el sistema de hacienda de la sierra norte. Este sector, estrechamente ligado a la conservadora Iglesia de Quito, había permanecido en permanente pugna con el Presidente Flores y su afán modernizador. Cordero buscó hábilmente una conexión directa con las autoridades de Roma, pasando por encima de las contingencias ligadas a los conservadores quiteños, en una estrategia que tenia el acuerdo implícito de la cúpula eclesiástica romana.

Aunque las ideas positivistas de orden y progreso habian articulado en su momento el acuerdo de conservadores ortodoxos y moderados en búsqueda del necesario desarrollo de la sociedad, enfrentada al progreso tecnológico y económico de la revolución industrial, la lucha por el poder político y económico era demasiado aguda en el Ecuador finisecular, y sus efectos se harian sentir con repercusiones internacionales inclusive.

El proceso de ampliación del Estado, de ejecución de obras públicas tecnificadas con necesidad de capitales extranjeros, que se había iniciado con la administración de García Moreno, chocaba a su vez con realidades nacionales complejas. La deuda interna habia sido motivo de agobio para los gobiernos ecuatorianos desde el comienzo de la banca moderna en 1860 , a la vez que el inicio de una relación de dependencia mutua. ${ }^{9}$ Los préstamos de los bancos de Guayaquil para financiar las obras del Estado y los gastos militares, ocupaban la mayor proporción del presupuesto nacional. Paralelamente, el riesgo del creciente endeudamiento del Estado con la banca guayaquileña y su secuela de control político preocupaba profundamente a Cordero. El poder legislativo, controlado por la fusión, no apoyó la gestión presidencial y empleó la beligerancia desde un comienzo.

Así, el pago de la deuda y la construcción del ferrocarril, enlazados por la urgencia de invertir capitales importantes, se convirtieron en el detonante de una ácida polémica y la toma de posíciones politicas a favor o en contra de los gobiernos progresistas. El jefe conservador Camilo Ponce había reclamado la conveniencia de seguir el procedimiento de Garcia Moreno en la construeción del ferrocarril, es decir, paulatinamente y con recursos ecuatorianos. Ponce aprovechó hábilmente el nacionalismo alimentado por la desconfianza hacia capitalistas extranjeros, y sepultó la posibilidad de reforma con otro argumento habitual de las élites, "el pais no está preparado para acometer grandes empresas". La oposición comenzó a hablar de una "nueva" deuda externa. Los liberales encontraron un motivo más para continuar su lucha contra los gobiernos progresistas, y en Panamá, Eloy Alfaro escribió el folleto "Deuda gordiana" 10

Al igual que su antecesor, Cordero estaba muy consciente de la importancia del crédito como instrumento fundamental para invertir y financiar las vias de comunicación en un país de escasos recursos. A pesar de una crisis financiera generalizada, pagó puntualmente los compromisos de la deuda externa, pero el Congreso de 1894 decidió suspender los desembolsos. Se argumentó entonces que debido a la recesión económica mundial, el sector comercial no estaba en condíciones de pagar impuestos sobre los derechos de aduana. El historiador Luis Robalino Dávila observa que la presión de los comerciantes sobre el Congreso condujo a interrumpir el servicio de la deuda como procedimiento inmediato para conjurar la disminución de sus beneficios. ${ }^{11}$

Aunque la Cámara de Diputados aprobó un proyecto de ferrocarril presentado por el Presidente al Congreso de 1894, el Senado prefirió dar fe a la argumentación de Camilo Ponce, cuya potente retórica amenazadora incidió en el rechazo de la iniciativa gubernamental. Este triunfo fue percibido por los conservadores como "la victoria de puro e ilustrado patriotismo (...) sobre un proyecto inconsulto, frenética y tenazmente sostenido por la vanidad de tres Gobiernos", una frase que aludia despectivamente a los Presidentes progresistas. ${ }^{12}$

El Congreso de 1894 se convirtió en una especie de termómetro del incendio político, atizado por una cerrada oposición durante un periodo extremadamente dificil para la formación de la sociedad moderna en el Ecuador. La Iglesia y los conservadores garcianos se irritaban con la moderación ideológica del

\footnotetext{
- El Presidente Flores, favorable al catolicismo liberal de vertiente europea, habia procurado impulsar una reforma del Estado en el dificil espacio dejado por el desajusté entre el clero ecuatoriano y la politica de apertura manejada por León XIII desde Roma. No tuwo éxito en sat emperio.

- Alexander Rodriguez, Linda. 1992. Las Fimanzas Pablicas en el Ecuador (1830-1940), Quito, Banco Central del Ecuador, p. 103.

10 Alfaro, Eloy. "Deuda gordiana", folleto escrito en Panama, con fecha 10 de febrero de 1892.

11 Robalino Divila, Luis. 1968, Onigerres del Ecuador de Hoy, VI, "Diex Anos de Civilismo", p. 608.

12 Gonzallez Paez. M. A. 1934. Memarias histíricus, Quito, Editorial Ecuatoriana, p. 72.

Revista de Historia, año 18, vols. 18-19, 2008-2009, pp, 79-91.
} 
progresismo y su inclinación a cierto liberalismo económico. Existian también razones personales en esta aversión. El poderoso Camilo Ponce no era ya el único interlocutor de la cúpula eclesiástica, debido a la relación directa que Cordero habia establecido con el arzobispo de Quito. Los ortodoxos de la capital habian perdido la exclusividad de la conexión con la Iglesia, "su" Iglesia, y no estaban dispuestos a ceder posiciones ante un político de provincia como Luis Cordero, por añadidura poeta en español y quichua, naturalista y filólogo.

La cultura de confrontación había encontrado un blanco excelente en la posición conservadora moderada. El Estado nacional tenia poco sentido para las élites contendientes, salvo en caso de conflicto de fronteras, en que el peligro del enemigo común generaba la colaboración y unidad de los adversarios en torno al gobierno. Este fue el caso durante el periodo de tensión derivado de la negativa del Congreso peruano a ratificar el tratado Herrera-García, que el Ecuador habia sancionado en 1890. La amenaza de un conflicto armado entre ambos paises precipitó el viaje del Enviado Extraordinario y Ministro Plenipotenciario del Perú, Emilio Bonifaz. Camilo Ponce aceptó el nombramiento de Ministro Plenipotenciario ad hoc para negociar un acuerdo con el representante peruano, y se mantuvo en permanente contacto con el Presidente Cordero mientras duraron las conversaciones diplomáticas.

Pero los liberales se impacientaban por la tolerancia del Presidente con la Iglesia. El violento debate en el Senado de 1894 respecto al político liberal Ángel Felicisimo López, elegido senador aunque había sido excomulgado en 1890 por el obispo de Portoviejo, Pedro Schumacher, llevó la situación a un extremo. Aunque elegido constitucionalmente, López no podía ejercer la senaduría por cuanto la excomunión implicaba la privación de los derechos cívicos. A fines del siglo XIX, tal descalificación era insostenible, y se justifica la razón anticlerical del liberalismo finisecular empeñado en la construcción del Estado nacional. La Iglesia no protegia únicamente sus privilegios, sino también un concepto de nacionalidad organizado en función de la universalidad del catolicismo romano, un principio que había sido consagrado por el Concordato garciano de 1863. El clero tradicionalista infundía en las mayorias el sentido de lo nacional dentro de una combinación peculiar de doble identidad: la identidad espiritual sometida a un soberano extranjero, el Papa, y la temporal, sujeta al poder político del Estado nacional, con predominio de la primera de ellas.

La corriente anticlerical fue solo uno de los factores que conducirian a la revolución liberal de 1895. Hacia fines de siglo, los intereses de los sectores modernizantes de costa y sierra, no necesariamente armónicos y no necesariamente coincidentes con la región de nacimiento, rechazaban continuar con el modelo económico-político de la hacienda tradicional, y confluian en una meta común, cual era establecer el estado secular, indispensable para el desarrollo material y cultural del pais, y para el acceso democrático al poder económico y politico. En este ambiente, las capas medias reclamaban firmemente sus oportunidades.

\section{LA "VENTA DE LA BANDERA" A CHILE}

El suceso denominado "venta de la bandera" llevó la agitación politica en el Ecuador a niveles pocas veces alcanzados, y permitió a la fusión conservadora-liberal construir una causa que unió a las regiones en torno a un objetivo común, cual era obtener la renuncia del Presidente Cordero.

A raiz de su enfrentamiento bélico con China, el Japón de la época buscaba fortalecer su marina de guerra y recurrió para ello a la Casa Flint, una empresa naviera situada en Nueva York, que sugirió la compra del crucero chileno "Esmeralda". Esta empresa conocía al sector comercial de Guayaquil, pues había participado en negociaciones para prolongar el ferrocarril desde el puerto principal hacia Quito. Chile estaba imposibilitado de hacer una venta directa por haber declarado su neutralidad en el conflicto, y Flint aconsejó pedir a un pais amigo, en este caso el Ecuador, que "adquiriese, a lo menos en apariencia, la propiedad de una nave, para transmitirla por cuenta de él a otra nación, en el puerto de Honolulu" 13 El asunto no era alquilar una bandera, puesto que la enseña no representaba un titulo de propiedad, $\mathrm{y}$ de haberlo necesitado, Chile hubiera podido escoger a cualquier pais para cubrirse con pabellón ajeno, sino que el "Esmeralda", navio de guerra, tuviese en regla sus papeles de procedencia en caso de una eventual revisión apoyada en el derecho internacional.

El tema no era ajeno al mundo de la época. En 1883, para evitar que su barco "Alajuela" apareciera como revolucionario, Eloy Alfaro habia izado una bandera colombiana, "como en 1894 el "Esmeralda" en Valparaiso se cubriria con la ecuatoriana", señala el historiador Wilfrido Loor. ${ }^{14}$ En el folleto antes

1s Cita del folleto atribuido \& Cárdenas, Alejandro, 1898. "El contrabando de la Esmeralds. Absolución al Gobierno", Quito, p. 9.

i4 Loor, Wilfrido. 1947. Eloy Alfaro, T.1, Quita, Editora Moderna. p. 131. 
mencionado, Alejandro Cárdenas cita una observación del eminente Andrés Bello: "Se ha vielto práctica universal la de navegar con diferentes pabellones, para disimular la nacionalidad, con la mira de evitar un ataque o llevar a cabo una perfidia".

La propuesta llegó el 16 de octubre de 1894 al entonces gobernador del Guayas, el ex Presidente progresista José Maria Plácido Caamaño, en un telegrama del cónsul de Ecuador en Washington, Modesto Solórzano. En esa época no existía comunicación cablegráfica directa entre el exterior y Quito, y los mensajes debían ser transcritos y enviados al gobierno central, un procedimiento que posibilitaba una modificación de contenidos. Caamaño envió el mensaje al Presidente Cordero, recomendando al mismo tiempo aceptar el trato.

Además de mantener antiguos vínculos politicos, de comercio y de intercambio profesional, Chile y Ecuador compartian un mismo y beligerante enemigo en cuestiones territoriales. Ambos paises se necesitaban, y Chile había quedado aislado después de su triunfo en la Guerra del Pacifico. Cuando las relaciones ecuatoriano-peruanas entraron en una fase de extrema tensión desde 1893, Chile sostuvo la causa ecuatoriana por la prensa y le vendió armamento en condiciones ventajosas. Al momento de hacer su proposición sobre el uso de la bandera en 1894, el Ecuador le debía una apreciable suma de dinero por los armamentos, y Chile presionó recordando esta deuda mediante telegrama, según indica Luis Cordero en su folleto aclarativo $A$ mis conciudadanos. Explicación circunstanciada de lo ocurrido en el odioso asunto del crucero de guerra "Esmeralda". 15 El Ecuador hubiese quedado en una situación muy incómoda ante un Estado aliado si su Presidente hubiera dado una negativa.

En telegrama del 20 de octubre de 1894, Cordero autorizó poner al "Esmeralda" bajo dominio ecuatoriano para trasladarlo a Honolulu desde el puerto de Chatam en el entonces Archipiélago de Colón, hoy Galápagos. En su folleto antes mencionado, $A$ mis Conciudadanos, el ex mandatario explicaba que tomó una decisión favorable a lo solicitado por Chile, basándose en el criterio de conocedores en Derecho Internacional, como el Dr. Pablo Herrera. En ese sentido anota: "Nada teniamos que ver nosotros con las naciones del Asia oriental, las que auin por la culta Europa se consideraban excluidas del derecho de gentes consuetudinario (...). Ia raza china era legalmente rechazada por varios pueblos civilizados, incluso el del Ecuador, (...) la guerra chino-japonesa no nos habia sido notificada en debida forma, y solo teniamos respecto a ella las noticias, no pocas veces inexactas, que suele dar el cable".

El 26 de octubre, el Presidente comisionó al cónsul ecuatoriano en Valparaíso, Luis A. Noguera, para estudiar el precio del posible negocio una vez cumplido el requisito anterior. Hasta alli, la situación no afectaba a la soberanía de ninguno de los países envueltos en la negociación. ${ }^{16}$ Pero el asunto se volvió embarazoso cuando el crucero zarpó de Valparaíso con bandera ecuatoriana, contrariamente a lo dispuesto por el Presidente. Habia una considerable suma de dinero en juego para los negociantes, y el contrato entre Ecuador y Japón se firmó en Nueva York el 23 de noviembre de 1894. La escritura de compraventa del "Esmeralda" fue otorgada el 30 de noviembre de 1894 en Valparaiso.

A comienzos de díciembre, la prensa de Guayaquil comenzó a informar en tono inquieto sobre las confusas circunstancias que rodeaban al uso de la bandera ecuatoriana. Uno de los primeros en dar la voz de alarma fue Luciano Coral, prestigioso periodista liberal del diario El Tiempo, el 4 de diciembre de 1894, tomando como base la información cablegráfica procedente de Valparaíso. ${ }^{17}$ Diarios del exterior como $\mathrm{La}$ Estrella de Panamá, El Mercurio de Santiago de Chile, El Comercio de Lima, informaban que el Ecuador había vendido un crucero al Japón, en guerra con China. Una amplia reunión cívica realizada el 8 de diciembre en el puerto principal, nombró una comisión investigadora. La intranquilidad de Guayaquil repercutió en Quito, donde violentas hojas sueltas de todos los colores políticos se tradujeron en manifestaciones populares. La protesta se volvió un mar incontenible. Desde su destierro en Centroamérica, Eloy Alfaro llamaba a la insurrección.

El gobierno trató de bajar el tono de la protesta, aunque mantuvo la reserva siguiendo el criterio del Consejo de Estado. El 14 de diciembre, el diario El Tiempo calificaba al tema como "crimen de lesa patria". El 15 de diciembre, Cordero emitió una proclama al pais, donde explicaba que luego de la exposición del Consejo de Estado sobre la inexistencia de hechos atentatorios contra la dignidad nacional, el Presidente

\footnotetext{
13 Cordero, Luis. 1896, "A mis conciudadanos. Explicación circunstanciada de lo ocurrido en el odioso asunto del crucero de guerra "Esmeralda". Cuenca, diciembre 2, p. 13.

in Cordero, Luis. A mis conciudadanos... p. 10.

i7 Coral, Luciano. 1934. "Quien ha comprado el Esmeralda?", en Articulos de Harodido, Guayaquil, s/p.
}

Revista de Historia, afio 18, vols. 18-19, 2008-2009, pp. 79-91. 
estimaba que no habia argumentos para sospechar del gobierno. ${ }^{18}$ Pero ese no era el punto, insistia El Tiempo el 20 de diciembre, porque no era el lucro lo verdaderamente importante, sino la honra de la nación.

El Presidente se reunió con dirigentes de la oposición para cruzar ideas sobre el manejo de la situación imperante. A fines de diciembre, el Presidente destituyó a los cónsules Solórzano y Noguera por considerar que su conducta habia sido irregular. En el folleto citado, A mis conciudadanos (1896), Luis Cordero deslinda toda responsabilidad en el fraudulento negocio y culpa a José María Plácido Caamaño, a Modesto Solórzano, Cónsul en Nueva York, y a Luis A. Noguera, Cónsul en Chile. Acusa a estos funcionarios de haber obrado con oscuros intereses de carácter económico. El historiador y diplomático chileno, Mario Barros van Buren, apunta que "según los documentos descubiertos, parientes del ex Presidente Caamaño se ganaron una comisión, que los intermediarios pagaron en libras esterlinas" 19

El 28 de diciembre de 1894, el Gabinete presidencial fírmó una Exposición, en la cual los Ministros Sarasti, Cárdenas y Espinosa aclaraban que el Ecuador no había comprado ni vendido el buque "Esmeralda", y anunciaban su resolución de pedir al agente diplomático en Chile que investigara la realidad de los hechos. Las protestas en contra del gobierno eran temerarias y maliciosas, decian los Ministros, porque los reclamos debian dirigirse contra los que habian intentado el fraude y la especulaciỏn valiéndose del pabellón nacional. ${ }^{20}$ El Gobernador Caamaño asumió la responsabilidad de los hechos, y el 4 de enero de 1895, Cordero pidió su renuncia. Caamaño la firmó el 11 de enero, y posteriormente viajó a España, donde se estableció sin mayor ostentación hasta su muerte en 1900. Más tarde, Cordero diría que "la proposición de venta del buque, hecha a Chile por el Sr. Caamaño, era harto diversa de la que le mandó a hacer el Ministro de Hacienda e interino de Guerra, y que precisamente de esa diversidad habian provenido todos los abusos" 21

El pais estaba convulsionado y nadie quería restarse al sentimiento patriótico exacerbado por la oposición. El Presidente, acosado por la agresividad de conservadores y liberales y por la efervescencia despertada, tenia preparado el texto de su dimisión desde el 14 de diciembre de 1894, en espera de presentarlo al Consejo de Estado cuando estuviese asegurada la transmisión constitucional al Vicepresidente de la República. El 4 de enero de 1895, el Comité investigador nombrado en Guayaquil publicó un informe donde pedia la renuncia del Presidente, "único medio de lavar la mancha del pabellón". 22 El manifiesto guayaquileño tuvo una resonancia inmediata en forma de círculos concéntricos. Además del Comité investigador, pidieron la dimisión de Cordero las sociedades Liberal Nacional, Círculo Liberal de Guayaquil, y Liberal y Conservadora de Quito. Las acusaciones al Presidente y su gabinete se extendieron por todo el país, provocando encendidas protestas de fervor patriótico y de rechazo a Cordero.

La "fustón" cerró el cerco de censura en torno al Presidente, y creó una atmósfera de escándalo en que cualquier intento de explicación era desoído. Cordero fue acusado de traición a la soberanía ecuatoriana, contrabando, peculado, tráfico de influencias, abuso de poder. No solo los notables guayaquileños y los radicales pedian la salida del mandatario. Los hacendados del norte, conservadores y liberales moderados, auspiciaron varios intentos de derrocamiento. Era "la 'fusión' en armas". 23

En el pais circulaban profusamente hojas sueltas y panfletos, con y sin firma, que pedian la renuncia del Presidente. El poder presidencial se habia debilitado en exceso y la situación se volvió indefendible. El 16 de abril de 1895, Luis Cordero hizo efectiva su renuncia ante el Consejo de Estado, que la aceptó de inmediato. El mismo dia, el Vicepresidente Vicente Lucio Salazar asumió como Encargado del Poder. Sería derrocado el 5 de junio de ese año por el movimiento a favor de Alfaro en Guayaquil, una fecha clásica para el Ecuador, llamada en lo sucesivo la Revolución Liberal.

El ánimo del ex Presidente Cordero acusó por largo tiempo el impacto de los golpes de la oposición, exaltada por un error que no tenía la magnitud atribuida, sino que habia sido un pretexto para quebrar la via progresista de gobierno. De regreso a su región natal, se dedicó a poner por escrito sus reflexiones sobre el episodio de la bandera y a esclarecer la participación de los distintos actores.

La Asamblea Nacional de 1896-1897, planteó el 7 de junio de 1897 una acusación judicial contra el ex Presidente Luis Cordero y su ministro de Hacienda, Alejandro Cárdenas, por simulación de un contrato contrario a la conveniencia de la República y supuesta malversación de fondos. El juicio pasó a la Corte

\footnotetext{
38 Condero, Luis. 1894. "Luis Cordero, Presidente de la República a los ecuatorianos": Quito, diciembre 12, AHBC/C, CHS 1893-1895, f. 70.

i9 Barros Van Buren, Mario. Op. cit. p. 96.

20 Sarasti, José Maria, Alejandro Cardenas, Roberto Espinosa. 1894. "Exposición del Ministerio", Quitch diciembre 28, AHBC, CH5 f. 73 .

21 Cordero, 1uis, 1897. Impugnación de unas observaciones hechas por D.J.R.M. en defensit del D.D.J.M.P. Caumaño, Cuenca, mayo 2, p. I7, Segundo folleto de Luis Cordero sobre la cuestión del crucero "Esmeralda".

22 Citado por Dávila, Robalino. 1968. Orígenes del Ecuador, ... p. 636.

23 J.L.R, (Jose Legohuir Raud o Rodas) Historia de la Repúbilica del Ecuador, I III (1876-1900), Quito, 1938 , I. III, p. 477.
} 
Suprema. El 6 de junio de 1898, el fiseal Adolfo Páez pidió el sobreseimiento a favor del los acusados, como un acto de estricta justicia, El 24 de octubre de ese año, la Corte Suprema resolvió por unanimidad declarar sin lugar la formación de causa contra el ex Presidente Luis Cordero y el ex Ministro Alejandro Cárdenas.

\section{LUIS CORDERO EN EL CENTENARIO DE LA INDEPENDENCLA DE CHILE}

El ambiente politico de Chile también se habia visto afectado por el episodio de la bandera. En 1897, la oposición al Presidente Jorge Montt, que gobernaba en el periodo de la República Parlamentaria, levantó su voz en el Congreso chileno para denunciar los acontecimientos en el Ecuador y expresar su solidaridad con el pueblo ecuatoriano. Pero la protesta no pasó a mayores. "Dada la cordura del pueblo chileno, la solidez de sus instituciones, la popularidad merecida del Presidente Montt y la habilidad del Ministro liberal Maclver, no se sufrió alli sino una agitación pasajera", escribe el historiador Legohuir al resumir los efectos políticos del episodio de la bandera en el pais del sur. ${ }^{24}$

Doce años más tarde, el gobierno liberal ecuatoriano haria justicia al ex mandatario. El Presidente Eloy Alfaro, a través de José Peralta, su Ministro de Relaciones Exteriores y figura señera del liberalismo ecuatoriano, ${ }^{25}$ ofreció a Luis Cordero el cargo de Enviado Extraordinario y Ministro Plenipotenciario del Ecuador para representar al pais en las fiestas del Centenario de la Independencia de Chile. En un gesto de generosidad política que no dejó de causar sorpresa, Luis Cordero aceptó esta misión.

Se trascribe a continuación la conceptuosa nota de Alfaro que formalizó la proposición ${ }^{26}$

\section{ELOY ALFARO, Presidente Constitucional de la República, a todos los que las presentes vieren: ;SALUD!}

Como el Gobierno del Ecuador ha sido invitado a concurrir, por medio de delegados especiales a las fiestas del Centenario de la Independencia de la República de Chile, y tiene plena confianza en el patriotismo, ilustración y competencia del Señor Doctor Don Luis Condero, ex Presidente de la República, le confiere, por estas credenciales, PLENOS PODERES para que represente al Gobierno en tan solemne ocasión, debiendo, al cumplir tan feliz encargo, significar al Gobierno Chileno la profunda simpatia del Poder Ejecutivo ecuatoriano por esa benemérita nación. Además, las presentes le habilitan también para gestionar y suscribir, ad referéndum, cualquier Pacto o Convención conducentes a estrechar más los vínculos que unen a los dos Paises.

En fe de lo cual firmo las presentes, selladas con las armas de la República y refrendadas por el Ministro Secretario de estado en el despacho de Relaciones exteriores, en Quito, a 29 de julio de 1910. ELOY ALFARO - J. PERALTA

El gobierno liberal buscaba reforzar lazos con un pais que habia dado pruebas concretas de amistad. En 1908, Chile habia vendido al Ecuador el buque de guerra "Almirante Williams", luego rebautizado como "Libertador Bolivar", el cual "durante muchos años seria la mejor de las naves de la marina ecuatoriana". 27 Chile también había enviado una misión naval-militar para ayudar al entrenamiento de las fuerzas ecuatorianas. Y en abril de 1910 habian llegado más armamentos y pertrechos para las fuerzas armadas ecuatorianas, traídos por otro "Esmeralda", esta vez un crucero liviano. ${ }^{28}$ El gobierno seleccionó con acierto la misión de Luis Cordero, para quien esta designación representaba un desagravio ante el Ecuador, Chile y el continente.

24 1.1. R. 1938. Historia de fa Repuiblias del Ecuadar, T. III (1876-1900), Quito, Imprenta del Cleto, p. 473.

24 Sobre esta importante figura histórica, ver Cárdenas Reyes, Maris Cristina. 2002. José Peralta y la Trayectoria del Liberatismo Ecaatoriano, Quito, Banco Central del Ecuador.

Is En Cordera, Luis. 1910. La Penipotencia Espedial del Ecuador en ef Primer Centenario de fa Independencia de Chile, Cuenca, pp. 4.5.

27. Cordero Crespo, Luis, 1999. Del surco a la cumbre. Biografia de Luis Cordeno, Cuenca, Monsalve Moreno Cla. p. 147.

28 Barros van Buren, Mario, 1966, Op. cit, p. 65.

Revista de Historia, año 18, vols. 18-19, 2008-2009, pp. 79-91. 
Una comunicación del Ministro Peralta, informó a Cordero sobre la comitiva oficial que lo acompañaria. ${ }^{29}$ Olmedo Alfaro era hijo del Presidente, lo que revela el interés de Alfaro en resaltar el grado de amistad entre ambos paises. A esta comitiva se sumaron, como adjuntos, tres hijos de Luis Cordero. El ex Presidente ya conocia Chile, país al que habia viajado en 1875 durante la presidencia de Federico Errázuriz Zañartu (1871-1876). En el desempeño de su función militar, Olmedo Alfaro también había viajado a Chile anteriormente, luego del gran terremoto de Valparaiso en 1906, y estaba vivamente interesado en reunirse con sus pares chilenos.

Quito, a 9 de agosto de 1910

\section{Sr. Ministro:}

El Sr: Ministro de Guerra y Marina, en oficio de la fecha, me dice lo que sigue:

"El Sr: Presidente de la República ha tenido a bien expedir, en esta fecha, el siguiente acuerdo: "Nombrar a los Señores Coronel Don Olmedo Alfaro y Capitán de Navio Don Francisco Fernández Madrid, en representación del Ejército y de la Marina nacionales, respectivamente, Miembros de la Comisión que el Gobierno del Ecuador envia ante el de la República de Chile, con motivo del primer Centenario de la Independencia de dicha Nación. - Comuniquese. Palacio Nacional \& rúbrica del Sr. Presidente, - El Ministro de Guerra y Marina, F.J. Martinez Aguirre". - Lo que tengo el honor de comunicar a U. para su conocimiento y fines consiguientes. - Dios y Libertad. - F. J. Martinez Aguirre.

De U. atento y seguro servidor,

\section{José Peralta}

\section{Al Sr. Dr. Don Luis Cordero, E. E. y Ministro Plenipotenciario en Misión Especial.}

La designación de Cordero fue recibida con beneplácito en todo el Ecuador, como lo prueban numerosas comunicaciones privadas y artículos de prensa en diarios de las principales ciudades del pais. EI tono de estos escritos era altamente elogioso hacia el ex mandatario, y no faltaron las menciones de afecto hacia Chile, "la nación más simpática y amiga que tenemos en el continente". 30 En Guayaquil, punto de embarque, fue objeto de honores especiales por parte de las autoridades alfaristas y de admiradores antes de emprender viaje el vapor "Aysén". En el transcurso del viaje, Cordero se enteró del fallecimiento del Presidente chileno Pedro Montt, y de inmediato hizo llegar sus condolencias al gobierno. Una vez en Chile, Cordero asistiria a las honras fúnebres del extinto mandatario en Valparaiso.

A su paso por el puerto del Callao, el importante diario limeño El Comercio dedicó un artículo especial a la misión ecuatoriana al Centenario de Chile, aunque no solo por respeto. Estaba en plena beligerancia la cuestión de limites que el Ecuador mantenia con el Perú desde los primeros años de su vida republicana, y el problema no daba señales de un final próximo en el tiempo. La delegación ecuatoriana fue cuidadosa en no pisar tierra peruana durante su largo trayecto, si bien Luis Cordero hizo gala de su talento diplomático y atendió a la prensa con la mayor cordialidad a bordo del barco.

En su diario de viaje, Luis Cordero relata que a mediados de 1909, el Ecuador había vivido momentos de gran incertidumbre a raiz de las presiones y roces internacionales relacionados con el problema limítrofe, los cuales habian tomado proporciones alarmantes a principios de 1910. El Presidente Alfaro había asumido personalmente el mando del ejército, y el célebre Arzobispo e historiador Federico González Suárez, había arengado a los soldados $\mathrm{y}$ al pueblo.

\footnotetext{
2* En Cordero, Luis. La Plenipotencia Especial del Ecuador (.... p. pp. 5-6,

\% Citado por Tbidem, p. 15 ,
} 
El ex Presidente era particularmente querido y respetado en el pais del sur, y fue recibido con honores y demostraciones de afecto en cada uno de los puertos chilenos que tocó la nave que lo llevaba a su destino. Los chilenos veian en Luis Cordero un intachable representante de aquella nación hermana a la que siempre habian acompañado en sus logros y en sus vicisitudes. Por su parte, Cordero manifestaba que al pisar tierra chilena, los ecuatorianos se sentian como en su propia patria. Honores oficiales, calor popular, desfiles cívicos, fiestas en salones señoriales, todo homenaje le fue tributado a la delegación ecuatoriana encabezada por el ex Presidente.

Luego de 18 dias de viaje, la delegación desembarcó en Valparaiso el 1 de septiembre de 1910. Con motivo de su llegada, se organizó en el puerto una manifestación masiva de apoyo al Ecuador, incluyendo la formación de una directiva para dar continuidad a este gesto. Un gran desfile tuvo lugar el 3 de septiembre, y estuvo encabezado simbólicamente por la Sociedad de Veteranos del 79. Al mismo tiempo, Cordero aprovechó las numerosas entrevistas en la prensa nacional para resaltar el avance aportado por el ferrocarril que Alfaro había inaugurado en 1908, uniendo asi Guayaquil y Quito, así como también las oportunidades que la próxima apertura del Canal de Panamá ofrecería a los puertos ecuatorianos. Igualmente ofreció su perspectiva favorable a una posible alianza entre Ecuador, Colombia y Venezuela, aunque en una relación de igualdad y sin regresar a la antigua Gran Colombia. ${ }^{31}$

La llegada a Santiago estuvo marcada por un nuevo duelo nacional -el fallecimíento de Elias Fernández Albano, sucesor del Presidente Montt - y la Misión ecuatoriana concurrió a las respectivas honras fúnebres. En calidad de Vicepresidente, Emiliano Figueroa dio continuidad al poder ejecutivo sin perturbación social alguna, actitud que causó admiración a Luis Cordero y su comitiva. Invitado por amigos chilenos, asistió a una sesión de la Convención Electoral, reunida para designar al ciudadano que ejerceria la Presidencia de la República. La proclamación de Ramón Barros Luco (1910-1915), por consenso de liberales y conservadores, fue un nuevo motivo de entusiasmo de los visitantes respecto al republicanismo chileno.

En todas las celebraciones, oficiales y privadas, la delegación ecuatoriana fue objeto de aplausos y vivas. El Dr. Cordero, excelente orador y literato, tuvo una lucida participación en los numerosos actos de celebración del Centenario chileno, en los que estuvieron presentes numerosas delegaciones diplomáticas de diferentes paises amigos, junto a destacadas personalidades del mundo intelectual de la época, como José Enrique Rodó, por entonces diplomático uruguayo, y Juan Zorrilla de San Martín, autor del poema "Tabaré". El coronel Olmedo Alfaro y el capitán Francisco Fernández, edecán de Luis Cordero, regresaron al Ecuador poco dias después de la celebración oficial del 18 de septiembre.

La Comisión del Centenario quiso cerrar con broche de oro la asistencia de la Misión ecuatoriana a las festividades del pais anfitrión, y organizó una cena en honor de Luis Cordero, representante del Ecuador, la cual tuvo lugar el 30 de septiembre. Asistió lo más selecto de la élite política chilena, en el lugar que era un verdadero símbolo de su poder, el Club de la Unión en Santiago, subrayando asi la importancia de los lazos de fraternidad y amistad entre Chile y Ecuador. ${ }^{32}$

Luis Cordero agradeció con un discurso que principalmente elogiaba la estabilidad del modelo republicano chileno. A su juicio un ejemplo de comportamiento civico y democrático que toda América debía emular.

II La pesibilidad de esta alianza permanece tatente en el horizonte de poder de Ecuador y Venezuela, principalmente.

32 Ver la nómina de los asistentes a este banquete en Cordero, Luis. La Plenipotencia Especial del Ecuador (..., p. pi 197, De esta obra se toman extractos de su discurso en dicho banquete, pp. 185-189.

Revista de Historia, ano 18, vols, 18-19, 2008-2009, pp. 79-91. 


\title{
DISCURSO DE LUIS CORDERO, DE AGRADECIMIENTO Y DESPEDIDA
}

\author{
(Extracto)
}

"Modelos como el de Chile, y el de otros pueblos dotados de laudable sensatez, son los que necesitamos en el continente para marchar con paso seguro por la senda del progreso. Naciones perfectamente organizadas, que de este modo se rigen, son las que deben dar prácticas lecciones a las que no logran todavía convalecer de la fiebre convulsiva que las aqueja, sangra y debilita". (...)

"Sorprende, Señores, y aún más, asombra y edifica, la gran lección de entrañable amor a las instituciones y de consumada prudencia que acabáis de dar, en este solo, en este preciso mes de los clásicos recuerdos y de las esplendorosas fiestas. Una imprevista fatalidad acumuló, como para turbarlas adrede, sucesos deplorables, que hubieran desconcertado a cualquier pueblo que no fuese Chile. Cae el benemérito Prócer que os gobernaba; lloráis por él; pero le dais inmediatamente sucesor no menos digno. Cae también éste; lamentáis la nueva péndida; pero pedís en el instante a la Ley otro ciudadano ilustre que os presida, y con él celebráis el gran natalicio de la Patria, sin perjuicio de honrar de manera conveniente a los esclarecidos difuntos que han pasado a lucir en las regiones excelsas de vuestra historia.

$Y$ luego, entre las galanas fiestas de vuestro Centenario, sobresale una, que es la más hermosa, que es verdaderamente grande. Os juntais en patriótica asamblea; ostentais los claros nombres de muchos personajes que pudieran gobernar cumplidamente el Estado; los presentáis en honrosa competencia; os empeñais, aparentemente discordes, en mantener invariables las nobles candidaturas; ipero aqui de la abnegación ciudadana! iAquí del extraordinario desprendimiento! Los prohombre proclamados eliminan generosamente sus nombres y suena de repente en todos los labios el bien aceptado de un eminente candidato; Lo aclaman todos los partidos; lo aplaude el pueblo y la República queda de plácemes".

A su vez, el diario santiaguino La Mañana, dirigido por el periodista y político Alfredo Irarrázaval, convocó y organizó una gran manifestación popular en honor de la República del Ecuador representada por el ex Presidente Luis Cordero. El Alcalde de Santiago y los regidores recibieron a Luis Cordero y a su comitiva, a la que se habia sumado desde un comienzo el Ministro del Ecuador en Chile, Rafael Elizalde, para luego conducirlos al balcón principal de la Casa Municipal de Santiago, desde donde presenciaron el impresionante homenaje.

En la noche del 1 de octubre, desfilaron miembros de todas las sociedades y corporaciones obreras de la capital, empleados y comerciantes, centros politicos, centros de estudiantes y de jóvenes, profesores primarios y secundarios, abogados, representantes de sociedades femeninas, de la prensa, veteranos de la guerra de 1879, representantes de las comunas rurales de la región. Numerosas familias santiaguinas contemplaron el desfile, que estuvo encabezado por el General (r) del Ejército de 1879, Estanislao del Canto. Las bandas y orfeones que integraban el desfile ejecutaron los himnos patrios de Ecuador y Chile, y los manifestantes llevaban enlazadas las respectivas banderas nacionales, avivando a ambos paises, a Luis Cordero y a Eloy Alfaro. La prensa capitalina cubrió en detalle el gran desfile, calificándolo como un espectáculo imponente en el que habian participado unas sesenta mil personas, cuya presencia refrendaba un rumbo común en el camino del progreso.

Otro significativo desfile tendria lugar en Valparaiso en la noche del 4 de octubre. Por la tarde de ese dia. Luis Cordero habia hecho entrega al Director General de la Armada de una bandera chilena de combate obsequiada por damas guayaquileñas al Buque-Escuela "General Baquedano", El 5 de octubre, la Misión ecuatoriana emprendió el viaje de regreso a su pais. A su llegada, el Presidente Alfaro y el Ministro Peralta hicieron llegar a Luis Cordero sendas notas de felicitación. 
De regreso a Cuenca, el 10 de enero de 1911 fue nombrado Rector de la Universidad del Azuay por el Congreso Nacional. Era un justo reconocimiento a quien habia puesto todo su empeño en la creación de este plantel y de la Universidad de Guayaquil, con el propósito de descentralizar la educación superior ecuatoriana. La muerte sorprendió a Luis Cordero en el ejercicio de su Rectorado, el 30 de enero de 1912, dos días después del asesinato en Quito del ex Presidente Eloy Alfaro y sus compañeros.

Los lazos de intercambio y amistad entre Ecuador y Chile se han ido construyendo con el empeño y la voluntad de figuras como las que participan en los acontecimientos aqui ofrecidos. Hoy como antes, ambos paises comparten aspiraciones e ideales que arrancan de muy atrás y nutren el espiritu republicano del siglo XIX. Estos valores compartidos han permitido que los intercambios culturales y económicos de las dos naciones vayan en constante aumento. La presencia de Luis Cordero en las celebraciones del primer centenario de la Independencia chilena se enmarca en este contexto, al tiempo que su trayectoria y visión política, presente en las luchas de su país y también en la relación con Chile, al que percibe y resalta como modelo republicano a seguir en el continente, requiere ser entendida desde la historia singular del Ecuador en los Andes. El agitado proceso de formación del Estado Nacional ecuatoriano, inmerso en una necesidad/rechazo a articularse con paises del exterior, y signado por la gravitación de las elites regionales y de caudillos de distinta procedencia, hace que Luis Cordero y su rico bagaje cultural nutrido por las culturas hispana e indigena, merezca una investigación de orientación comparativa que contribuiria a iluminar procesos mayores en los que un pasado, no demasiado lejano, se mantiene activo en las heterogéneas sociedades latinoamericanas de hoy.

\section{FUENTES Y BIBLIOGRAFIA}

\section{FUENTES ÉDITAS}

\section{Archivo Histórico del Banco Central del Ecuador Sucursal Cuenca (AHBC/C), Colecciones de Hojas Sueltas (CHS):}

"Luis Cordero, Presidente de la República a los ecuatorianos". Imprenta del Gobierno. Quito, diciembre 12 de 1894. AHBC/C, CHS 1893-1895, f. 70.

José Maria Sarasti, Alejandro Cárdenas, Roberto Espinosa. "Exposición del Ministerio". S. p. i. Quito, diciembre 28 de 1894. AHBC/C, CHS 1893-1895, f. 73.

\section{Folletos y artículos de periódico:}

Alfaro, Eloy. 1959. "Deuda Gordiana", Segunda edición corregida y aumentada por el autor, Quito, Imprenta Nacional, 1895. En Obras Escogidas, Tomo II, Guayaquil, Editorial Viento del Pueblo, pp. 5-64.

Cárdenas, Alejandro. 1898. El contrabando de la Esmeralda. Absolución al Gobierno, Quito, Imprenta "La Novedad".

Coral, Luciano, 1934. Arriculos de Haroldo (Luciano Coral), publicados en Guayaquil en 1894-98, Guayaquil, IImp. de "El Tiempo".

Cordero, Luis. 1896. A mis conciudadanos. Explicación circunstanciada de lo ocurrido en el odioso asunto del crucero de guerra "Esmeralda", Cuenca, Imprenta de José María Montesinos e Hijos.

Cordero, Luis. 1897. "Impugnación de unas observaciones hechas por D.J.R.M. en defensa del D.D.J.M.P. Caamaño", Cuenca, Impreso por M. Vintimilla C., mayo 2, p. 17.

Cordero, Luis. 1910. La Plenipotencia Especial del Ecuador en el Primer Centenario de la Independencia de Chile, Cuenca, Tip. de la Universidad. 


\section{BIBLIOGRAFIA CITADA}

Alexander Rodriguez, Linda. 1992. Las Finanzas Públicas en el Ecuador (1830-1940), Quito, Banco Central del Ecuador.

Barros van Buren, Mario. 1976. La Misión Eastman en el Ecuador, Quito, Casa de la Cultura Ecuatoriana. Cárdenas Reyes, Maria Cristina. 2002. José Peralta y la Trayectoria del Liberalismo Ecuatoriano, Quito, Banco Central del Ecuador.

Cárdenas Reyes, María Cristina. 2006. Región y Estado Nacional. El Progresismo Azuayo del Siglo XZX (18401895), Quito, Academia Nacional de Historia, Ecuador/Universidad Pablo de Olavide.

Cordero Crespo, Luis. 1999. Del surco a la cumbre. Biografia de Luis Cordero, Cuenca, Monsalve Moreno Cia.

González Páez, M. A., 1934. Memorias históricas. Editorial Ecuatoriana, Quito.

J.L.R. (José Legohuir Raud o Rodas), 1938. Historia de la República del Ecuador, T. III (1876-1900), Quito Imprenta del Clero.

Loor, Wilfrido. 1947. Eloy Alfaro, T. L., Quito, Editora Moderna.

Palomeque, Silvia. 1990. Cuenca en el siglo XIX, La articulación de una región, Quito, FLACSO/Abya Yala. Robalino Dávila, Luis, 1968. Origenes del Ecuador de Hoy, T. V1, "Diez Años de Civilismo", Puebla, Ed. J. M. Cajica.

Tobar Donoso, Julio, 1925. Desarnollo constitucional del Ecuador, Quito, Tip. y Encuad. de "La Prensa Católica".

\section{OTROS ESCRITOS DE LUIS CORDERO (Una breve selección)}

Adiós del indio: pequeña composición poética, escrita en idioma quichua, para deplorar, de algun modo, la desdicha de una gran parte de los indios que en el pais se llaman conciertos / Prosa y verso / Impreso por Andrés Cordero, Cuenca, 1875.

Excursión a Gualaquiza: observaciones sobre el camino que conduce a esta colonia, sobre el estado actual y futuro de ella, y en la clasificación y usos de algunas plantas vistas por el autor en su pequeño viaje. S.e., Cuenca, 1875 .

Cultivo de las Quinas. Traducción hecha por Luis Cordero de algunos capitulos interesantes de la obra intitulada 'Nowvelles Etudes sur les Quinquinas'; escritas por el ilustre botánico calombiano Don J. Triana. Impreso por Antonio Cueva, Cuenca, 1877.

Recuerdos Patrióticos de 1883. Discursos, poesias y otras producciones de Luis Condero, dadas durante los ocho meses de permanencia en el Gobierno Provisional de la República. Fundición de Tipos de M. Rivadeneira, Quito, 1883.

Cushiquillca: Composición quichua en que un indio del Azuay celebra la cesantía de los diezmeros. Impreso por Andrés Cordero, Cuenca, 1884.

Rinimi llacta! Composición quichua en que un indio del Azuay lamenta sus desventuras. Reimpreso por Andrés Cordero, Cuenca, 1884.

Nuestra cuestión de limites. Universidad de Cuenca, Cuenca, 3a. edición, 1903.

"Alocución de Luis Cordero al posesionarse del Rectorado de la Universidad azuaya", Revista Cientifica y Litenaria de la Universidad del Azuay, Serie III, N 1. Cuenca, 1911, pp. 7-12.

Enumeración botánica de las principales plantas, asi útiles como nocivas, indigenas o aclimatadas, que se dan en las provincias del Azuay y del Cañar de la República del Ecuador. Afrodisio Aguado, Madrid, 2a. edición, 1950.

Diccionario quichua-español español-quichua. Casa de la Cultura Ecuatoriana Benjamin Carrión. Quito, 1955. 